B R A G A N T I A

Revista Científica do Instituto Agronômico do Estado de São Paulo

Vol. 32

Campinas, abril de 1973

N. ${ }^{\circ} 7$

\title{
DETERMINAÇÃO DE CARGAS ELÉTRICAS EM SOLOS ( $\left.{ }^{1}\right)$
}

\author{
Bernardo van RaIJ (2), Seção de Pedologia, Instituto Agronômico
}

\section{SINOPSE}

As cargas elétricas dos solos estudados variaram em função da concentração da solução, do $\mathrm{pH}$ e da valência dos ions trocáveis. Optou-se pela Jeterminação das cargas em condições de equilíbrio relativamente a pH e conzentração da solução, omitindo-se uma lavagem total do excesso de solução salina. Cargas positivas e negativas variaram com o sal empregado, para amostras do horizonte $\mathrm{B}_{2}$ de um Latossolo Roxo e de uma Terra Roxa Estruturada, aumentando na ordem $\mathrm{NaCl}<\mathrm{CaCl}_{2}<\mathrm{MgSO}_{4}$, para determinados valores de concentração e $\mathrm{pH}$.

\section{1 -- INTRODUÇÃO}

Além de cargas elétricas negativas, responsáveis pela retenção de catíons trocáveis, podem ocorrer em solos, em determinadas condições, cargas elétricas positivas, responsáveis pela retenção de aníons trocáveis.

A existência de propriedades de troca de aníons, além das de troca de catíons em solos, foi demonstrada por Mattson já em 1927 (7). Posteriormente, Schofield (13) descreveu um método para a determinação de cargas positivas e negativas em solos, que serviu de base para a maior parte dos métodos que surgiram depois.

(1) Recebido para publicação em 20 de maio de 1972.

(2) Com bolsa de suplementação do CNPq. 
Contudo, a predominância de cargas negativas em solos de clima temperado, acompanhada da virtual ausência de cargas positivas, fez com que o interesse pelo estudo de cargas elétricas ficasse praticamente limitado, para os solos daquelas regiōes, ao estudo da troca de catíons. Os trabalhos que tratam de cargas positivas e troca de aníons em solos são realizados, em geral, com amostras de regiões tropicais $(4,11,15)$.

Em trabalho relatado recentemente são apresentados resultaăos de cargas elétricas para alguns solos do Estado de São Paulo, com ênfase à variação da carga líquida dos solos (11) .

Neste trabalho é relatado um estudo sobre cargas positivas e negativas nos mesmos solos, com ênfase ao método de determinaçấo e à variação das cargas positivas e negativas.

\section{2 - MATERIAL E METODO}

Foram utilizadas amostras dos horizontes $A_{k}$ e $\mathbf{B}_{2}$ de três unidades de solos do Estado de São Paulo. As amostras de um Latossolo Roxo (T 4108 e T 4109) e de uma Terra Roxa Estruturada ( $P$ 1012a e 1012c) foram coletadas em Ribeirão Preto, enquanto amostras de um Latossolo Vermelho Amarelo húmico ( $\mathrm{T} 4119$ e 4120) foram coletadas em Campinas. Neste trabalho essas três unidades de solos serão apresentadas respectivamente por LR, TE e LH. Em outro trabalho, em que os solos mencionados foram denominados, respectivamente, Acrorthox, Tropudalf e Acrohumox, são apresentadas as características físicas, químicas € mineralógicas (11).

Todas as amostras foram secas ao ar e passadas em peneira de $2 \mathrm{~mm}$. Para as determinações de cargas elétricas, em que foram pesados apenas $2 \mathrm{~g}$ de terra, foram utilizadas subamostras homogenizadas e passadas em peneira de $0,71 \mathrm{~mm}$.

A determinação de cargas elétricas em solos pode ser dividida em quatro etapas distintas, coincidentes com as quatro etapas de determinação da capacidade de troca de catíons, que são: a) saturação do solo com íons-índice escolhidos para determinar 
as cargas elétricas, através de lavagem com solução salina, em condições especificadas de concentração e pH; b) remoção do excesso da solução salina; c) extração dos íons-índice com outra solução salina; d) determinação analítica dos íons-índice.

A seguir é descrita a metodologia proposta para a determina.. ção de cargas elétricas em solos, baseada em grande parte no trabalho de Schofield (13), com algumas modificações introduzidas durante a realização deste trabalho no laboratório. Foram feitas determinações com soluções de $\mathrm{NaCl}, \mathrm{MgSO}_{4}$ e $\mathrm{CaCl}_{2}$.

a) Satursçâo dos solos com íons-índice - Colocou-se o equivalente a $2 \mathrm{~g}$ de terra fina seca na estufa em um copo de $100 \mathrm{ml}$ e adicionaram-se $50 \mathrm{ml}$ de $\mathrm{NaCl} 0,2 \mathrm{~N}$. O pH foi ajustado com solução de $\mathrm{HCl}$ ou $\mathrm{NaOH}$ para o valor desejado, fazendo-se leituras em potenciômetro, após homogenizar a suspensão. Após uma. noite de contacto agitou-se novamente a suspensão e leu-se o pH, reajustando-o quando necessário. A suspensão foi transferida, em duas etapas, para tubo de centrífuga pré-tarado, de $50 \mathrm{ml}$, e a solução de $\mathrm{NaCl} 0,2 \mathrm{~N}$ separada por centrifugação. Em seguida o solo foi lavado três vezes com volume de $40 \mathrm{ml}$ da solução $0,2 N$ cio sal usado para saturação (cinco vezes nos casos em que $0,2 \mathrm{~N}$ foi escolhida como concentração final) .

b) Remocão do excesso de solução salina - A amostra foi lavada cinco vezes com $40 \mathrm{ml}$ de solução mais diluída do mesmo sal utilizado para saturar o solo (neste trabalho foram empregadas soluções $0,1,0,01$ e $0,001 N$ ) . O pH foi reajustado com $\mathrm{NaOH}$ ou $\mathrm{HCl}$ antes das duas ou três últimas lavagens. Após a última centrifugação, o tubo de centrífuga foi drenado e imediatamente pesado. Determinou-se $\circ \mathrm{pH}$ do último extrato.

c) Extração dos ínns.índice - A amostra foi extraída cinco vezes com $20 \mathrm{ml}$ de solução $0,5 \mathrm{~N}$ de $\mathrm{NH}_{4} \mathrm{NO}_{3}$, no caso de solos que haviam sido tratados com $\mathrm{NaCl}$, e com solução de $0,5 \mathrm{~N}$ de $\mathrm{NaNO}_{3}$, no caso de solos que haviam sido tratados com $\mathrm{CaCl}_{2}$ e $\mathrm{MgSO}_{4}$. A extração foi feita por centrifugação, e os extratos coletados em balão volumétrico de $100 \mathrm{ml}$. Alíquotas foram retiradas para as determinações de $\mathrm{Na}^{+}, \mathrm{Ca}^{2+}, \mathrm{Cl}^{-*}$ e $\mathrm{SO}_{4}{ }^{2-}$. 
d) Determinação analítica dos íons-índices - Alíquotas de $20 \mathrm{ml}$ dos extratos que continham sódio foram diluídas a $100 \mathrm{ml}$ com água destilada. A leitura foi feita em fotômetro de chama a $589 \mathrm{~nm}$, e o teor de sódio determinado através de curva-padrão obtida com soluções que continham teores conhecidos de $\mathrm{NaCl}$ e a solução extratora de $\mathrm{NH}_{4} \mathrm{NO}_{3} 0,5 \mathrm{~N}$ na proporção de $20 \mathrm{mI}$ por $100 \mathrm{ml}$.

Cálcio e magnésio foram determinados pelo método do EDTA. Como somente havia ou cálcio ou magnésio nos extratos a serem analisados, a mesma marcha analítica pôde ser utilizada para os dois elementos. Os reagentes empregados foram descritos em trabalho anterior (9). Um alíquota de 25 ou $50 \mathrm{ml}$ do extrato que encerrava cálcio ou magnésio foi colocada em frasco Erlenmeyer de $250 \mathrm{ml}$, e o volume completado até aproximadamente $100 \mathrm{ml}$ com água destilada. Acrescentaram-se $5 \mathrm{ml}$ de solução-tampão pH 10, 6 gotas de solução do indicador preto de eriocromo $\mathrm{T}$ e uma pitada (cerca de $5 \mathrm{mg}$ ) de dietilditiocárbamato de sódio. Titulou-se até viragem de vermelho para azul.

O íon cloreto foi determinado por titulação potenciométrica, usando elétrodo de $\mathrm{Ag}-\mathrm{AgCl}$ como indicador e elétrodo de quinidrona como referência (6). Elétrodos de $\mathrm{Ag}-\mathrm{AgCl}$ foram preparados por deposição eletrolítica de cloreto de prata em um fio de platina, seguindo a descrição de Brown (3). O fio de platina foi selado na extremidade de um pedaço de tubo de vidro amolecido à chama. O elétrodo de quinidrona consistiu de um pedaço de fio de platina, também selado na extremidade de um tubo de vidro, e imerso em uma solução tampão de biftalato de potássio $\left(1,02 \mathrm{~g}\right.$ de biftalato de potássio e $14,8 \mathrm{ml}$ de $\left.\mathrm{H}_{2} \mathrm{SO}_{4} 0,1 \mathrm{~N} \mathrm{em} 100 \mathrm{ml}\right)$ saturada em quinidrona e contida em uma cuveta de $10 \mathrm{ml}$. Ambos os elétrodos foram ligados a um medidor de $\mathrm{pH}$ através de fios de cobre mergulhados em mercúrio colocado no interior dos elétrodos. Uma alíquota de $50 \mathrm{ml}$ do extrato que continha cloreto foi transferida para copo de $150 \mathrm{ml}$, e esta solução foi ligada ao elétrodo de quinidrona através de uma ponte de ágar saturada de $\mathrm{KNO}_{3}$. O elétrodo de $\mathrm{Ag}-\mathrm{AgCl}$ foi também mergulhado no extrato. Para cada série de titulações tomou-se uma alíquota de $50 \mathrm{ml}$ da solução extratora, acrescentaram-se $5 \mathrm{ml}$ de solução que continha $101 \mathrm{~g}$ de $\mathrm{KNO}_{3}$ e $62 \mathrm{ml}$ de $\mathrm{HNO}_{3}$ concentrado em 1 litro 
de solução, algumas gotas de suspensão de $\mathrm{AgCl}$, e anotou-se a leitura no aparelho de $\mathrm{pH}$. Ao titular extratos de solos, adicionaram-se $5 \mathrm{ml}$ da solução de $\mathrm{KNO}_{3}$ e $\mathrm{HNO}_{3}$ e titulou-se com solução de $\mathrm{AgNO}_{3}, 0,01 \mathrm{~N}$ até o potencial lido no aparelho de $\mathrm{pH}$ coincidir com a leitura da prova em branco. A solução foi agitada continuamente com agitador magnético durante a titulação.

O íon sulfato foi determinado como sulfato de bário, por método turbidimétrico (1). A uma alíquota de $20 \mathrm{ml}$ do extrato que continha sulfato acrescentaram-se $2 \mathrm{ml}$ de uma solução de $\mathrm{HCl}$ $6 \mathrm{~N}$ que encerrava $20 \mathrm{ppm}$ de $\mathrm{S}$ na forma de $\mathrm{K}_{2} \mathrm{SO}_{4}$. Adicionou-se, imediatamente em seguida, $1 \mathrm{~g}$ de $\mathrm{BaCl}_{2} . \mathrm{H}_{2} \mathrm{O}$ (cristais de 20-60-mesh) e deixou-se a solução em repouso por 1 minuto. Em seguida agitou-se o frasco até dissolução dos cristais. Em um período de 2 a 8 minutos após, fez-se a leitura em espectrofotômetro a $420 \mathrm{~nm}$. A curva-padrão foi obtida com soluções que continham quantidades conhecidas de $\mathrm{K}_{2} \mathrm{SO}_{4}$ e a solução extratora.

As cargas elétricas dos solos foram calculadas como sendo a quantidade total de íons extraída dos solos menos a quantidade de íons contida na solução em equilíbrio. Este último valor foi obtido pesando-se os tubos de centrífuga no início e no final da operação de saturação do solo.

\section{3 - RESULTADOS}

No quadro 1 são apresentados alguns resultados obtidos após pré-tratamento de solos com solução de acetato de sódio $1 N$, $\mathrm{pH} 4,8$. A escolha desse $\mathrm{pH}$ foi arbitrária, tendo-se procurado sstabelecer condições em que os solos apresentassem cargas positivas e negativas. Após tratar $10 \mathrm{~g}$ de solo com $100 \mathrm{ml}$ de acetato de sódio, filtrou-se a suspensão através de funil de Buchner a vácuo. Adicionaram-se ao solo, em pequenos incrementos, $100 \mathrm{ml}$ de solução de $\mathrm{CaCl}_{2}, 1 \mathrm{~N}$ ou de $\mathrm{MgSO}_{4} 1 \mathrm{~N}$. O excesso de cloreto de cálcio foi removido com uma lavagem de $20 \mathrm{ml}$ de água e quatro lavagens com etanol a $95 \%$. O excesso de sulfato de magnésio não pôde ser removido com etanol, por ser o sal bastante insolúvel neste solvente, e, por tal razão, foram utilizadas cinco lavagens com $20 \mathrm{ml}$ de água. Os íons foram extraídos com solução de $\mathrm{NaNO}_{3} 1 \mathrm{~N}$ e determinados pelos métodos descritos. 


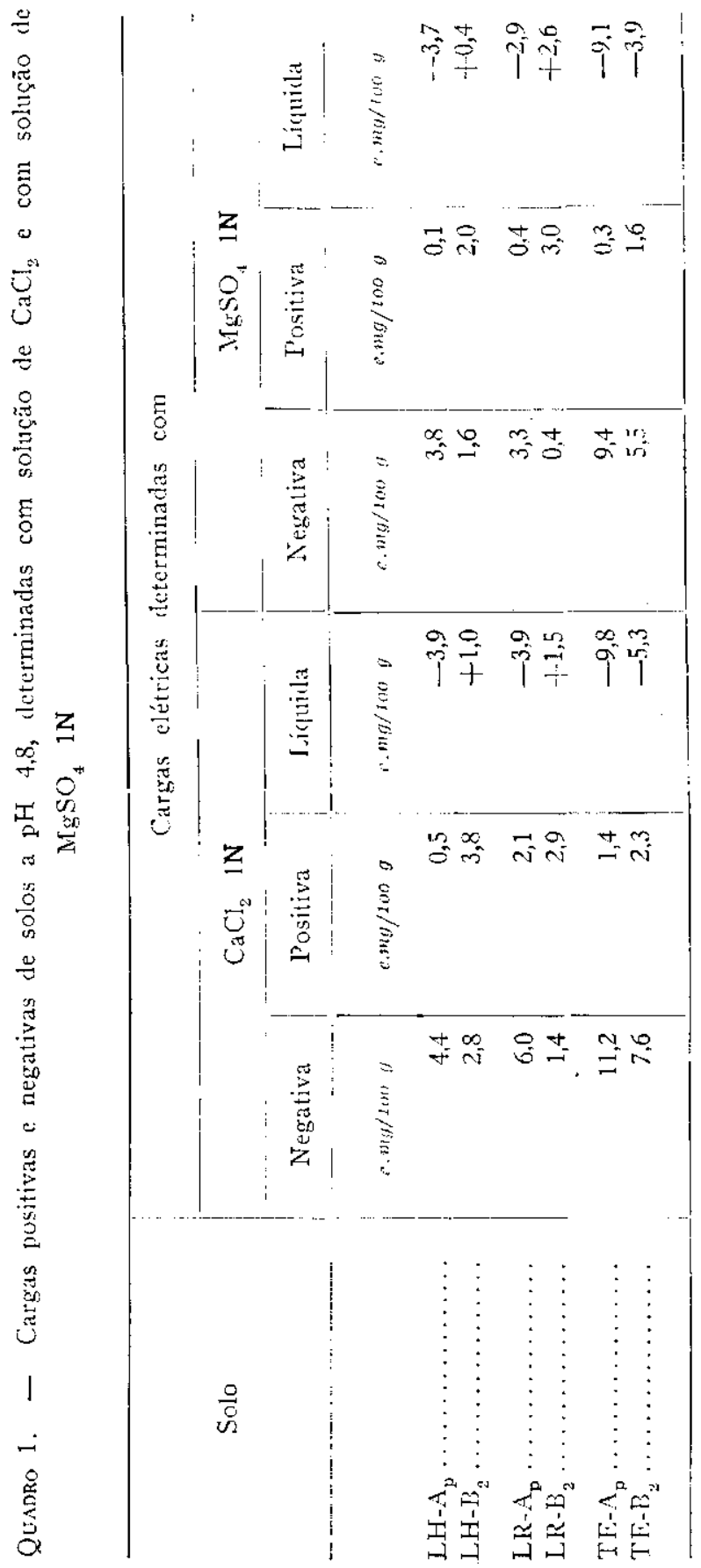


No quadro 2 são apresentados os dados de uma experiência realizada com o intuito de estudar a técnica de lavagem do excesso de sal. As determinações foram feitas como é descrito.

Q) Vadro 2. - Lifluência da lavagem do excesso de soluçäo salina. nas cargas elétricas de lima amostra do horizonte $\mathrm{B}_{2}$ de um Latossolo Roxo, detcrminadas com soluçāo de $\mathrm{CaCl}_{2}$ 1 $\mathrm{N}$ a $\mathrm{pH} 4,8$

\begin{tabular}{|c|c|c|c|}
\hline \multirow[b]{2}{*}{ Técnica de lavagem yara $10 \mathrm{~g}$ de solo } & \multicolumn{3}{|c|}{ Carga clétrica } \\
\hline & Negativa & Positiva & T.íçuida \\
\hline & $\mathrm{crmg/100 \textrm {g }}$ & $e . m g / 100 g$ & $e . m g / 100 g$ \\
\hline inl te $\mathrm{H}_{2} \mathrm{O} \div 4 \times 10 \mathrm{ml}$ de etanol $\ldots$. & 1,4 & 2,9 & $+1,5$ \\
\hline ml de $\mathrm{H}_{2}^{2} \mathrm{O} \perp 10 \times 20 \mathrm{ml}$ de ctanol $\ldots$. & 0,9 & 2.8 & $+1,8$ \\
\hline $20 \mathrm{ml} \mathrm{de} \mathrm{CaCl}_{2}, 0,1 \mathbf{N} \ldots \ldots \ldots$ & 2,1 & 3,6 & $+1, \tilde{5}$ \\
\hline $20 \mathrm{ml}$ de $\mathrm{CaCl}_{2}, 0,01 \mathrm{~N} \ldots \ldots \ldots \ldots$ & 0,5 & 2,0 & $-1,7$ \\
\hline$\times 20 \mathrm{ml}$ de $\mathrm{CaCl}_{22} 0,001 \mathrm{~N} \ldots \ldots \ldots \ldots$ & 0,3 & 1,5 & $+1,3$ \\
\hline $\begin{array}{r}\mathrm{ml} \text { de } \mathrm{H}_{2} \mathrm{O}-10 \times 20 \mathrm{ml} \text { de } \mathrm{CaCl}_{2} \\
0,001 \mathrm{~N}\end{array}$ & 0,2 & 1,4 & +1.3 \\
\hline 20 ml de $\mathrm{H}_{2} \mathrm{O}-10 \times 20 \mathrm{~m} 1$ de $\mathrm{H}_{0} \mathrm{O} \ldots$ & 0,1 & 1,2 & $+1,1$ \\
\hline
\end{tabular}

Após examinar os dados dos quadros 1 e 2 e tendo em conta informações dadas na literatura, foi desenvolvida a marcha analítica apresentada em Materiais e Métodos. Resultados para cargas positivas e negativas são apresentados na figura 1 . Convém frisar que no método utilizado evitou-se a lavagem completa do excesso da solução salina usada para saturação do solo, fazendo-se, em alternativa, lavagem com solução salina de concentração pre-determinada, até obtenção de equilíbrio. Verificou-se, ainda, ser mais conveniente o uso de centrífuga, já que com esta técnica as condições para obtenção de equilíbrio são mais favoráveis do que no caso do uso de filtros. 


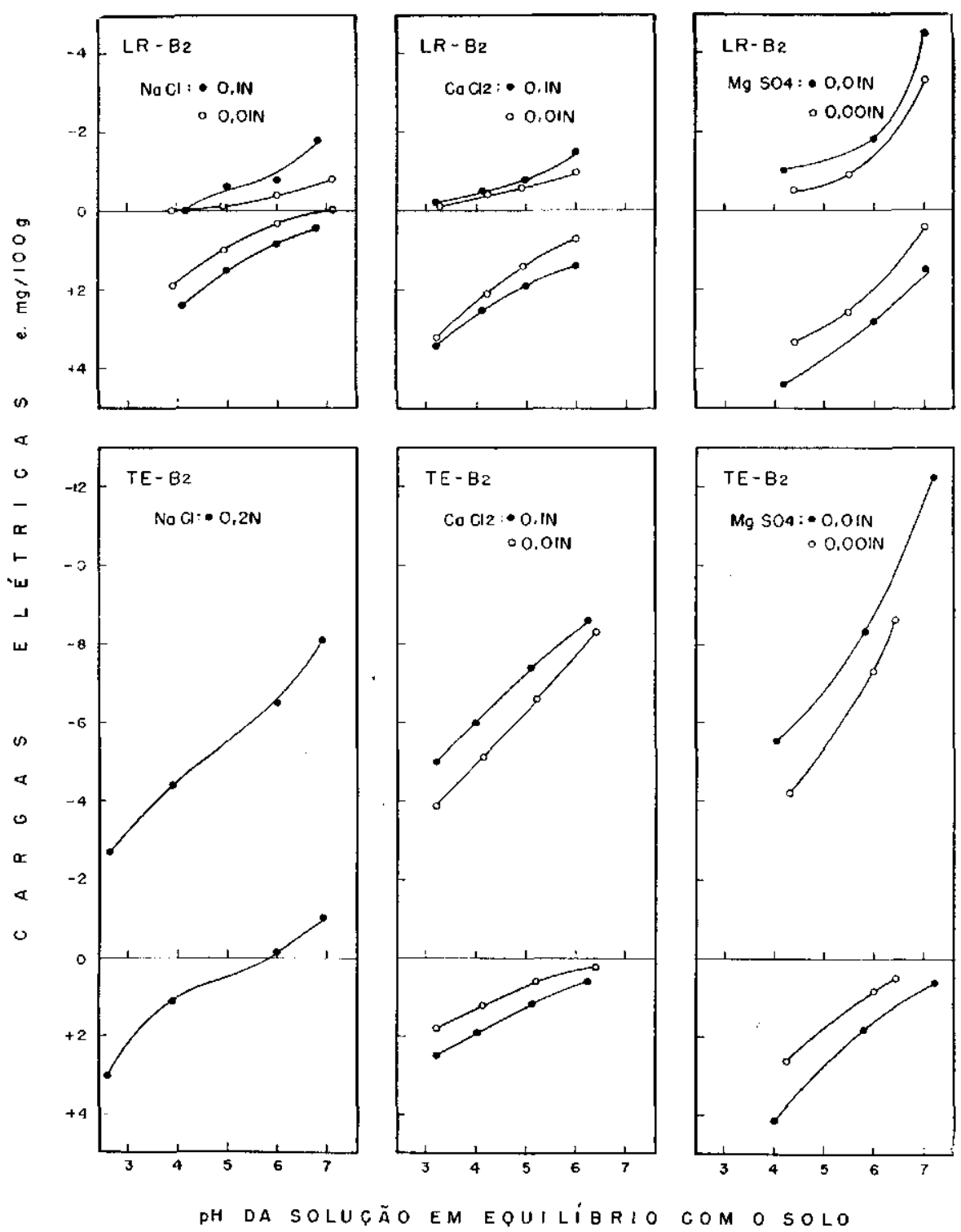

FIgURA 1. - Cargas elétricas positivas e negrativas, do horizonte $B_{2}$ de um Latos olo Roxo e de uma Terra Roxal Estruturada, deterninadas com solusoócs de $\mathrm{NaCl}, \mathrm{CaCl}_{2}$ e $\mathrm{MgSO}_{4}$. 


\section{4 - DISCUSS̃̃O}

Os resultados de cargas elétricas positivas, negativas e líquidas, determinadas com $\mathrm{CaCl}_{3}$ e $\mathrm{MgSO}_{4}$ para diversos solos (quadro 1), são bastante discrepantes. Os resultados mais elevados obtidos com $\mathrm{CaCl}_{2}$, ao contrário do que seria de esperar, permitem levantar duas suspeitas comuns nos trabalhos dessa natureza: má lavagem do excesso de cloreto de cálcio e/ou hidrólise dos íons $\mathrm{Mg}^{2+} \mathrm{e} \mathrm{SO}_{4}{ }^{2-}$ na lavagem com água.

A influência da etapa de lavagem nos resultados de cargas elétricas é claramente demonstrada no quadro 2. Há uma série de trabalhos que tratam do assunto da lavagem do excesso de sal em determinações da capacidade de troca de catíons que corroboram as informaçōes inferidas do quadro 2, isto é, o fato de os resultados de cargas serem função da técnica de lavagem $(2,5$, $8,12,14)$.

Em estudo recente, com as amostras de solo utilizadas neste trabalho, foi demonstrada a variabilidade da carga elétrica líquida dos solos com o pH e a concentração da solução em que foi estabelecido o equilíbrio. A variação dos resultados estava de acordo com previsões feitas pela teoria da dupla camada elétrica. Essa teoria prevê que, para superfícies sólidas em que o ín $\mathbf{H}^{+}$ funciona como determinador de potencial - provavelmente $o$ caso dos solos que estão sendo estudados - as cargas superficiais são influenciadas pela constante dielétrica da solução em equilíbrio com o solo, pela temperatura da suspensão, pela concentração e valência do íon trocável e pelo $\mathrm{pH}$ da solução $(\mathbf{1 0}, \mathbf{1 1})$. Note-se que a lavagem do excesso de sal com água ou soluções salinas diluídas altera a concentração, e que a lavagem com solvente orgânico como o etanol altera a constante dielétrica do meio, além de alterar a concentração da solução em contacto com o solo. Conseqüentemente, as cargas superficiais do solo são alteradas e a sua variação com a técnica de lavagem (quadro 2) não é surpreendente.

É importante, neste ponto, definir o que se almeja ao procurar um método adequado para a determinação das cargas elétricas em solos. Em determinações da capacidade de troca de 
catíons é comum falar em "erros" introduzidos pela lavagem do excesso de sal. Uma premissa muitas vezes admitida em tais casos é que a capacidade de troca de catíns é um número relativamente constante para cada solo. No entanto, para solos de clima tropical úmido é mais correto admitir que as cargas elétricas na superfície dos solos variam conforme as condições em que são determinadas. Portanto, as condições de determinação das cargas elétricas devem ser bem estabelecidas, como é o caso do método proposto neste trabalho, em que é definido o equilíbrio com relação à concentração salina e ao $\mathrm{pH}$.

Os resultados apresentados na figura 1, para amostras do

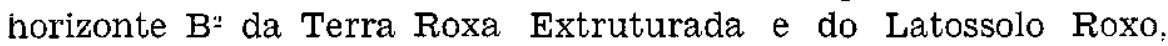
foram obtidos pela metodologia apresentada neste trabalho. Os fatores que influem nas cargas elétricas dos solos foram mantidos constantes ou medidos. Para cada sal empregado para as determinações das cargas elétricas fez-se variar os valores de $\mathrm{pH}$ e concentração das soluções. Como é muito difícil predeterminar o pH das suspensões dos solos sem usar soluções-tampão, o seu valor foi ajustado aproximadamente, e o $\mathrm{pH}$ do último extrato (a solução mais diluída) tomado como o pH de equilíbrio. Convém ressaitar que o método descrito neste trabalho, embora sendo basicamente correto, é bastante trabalhoso. O emprego de soluções-tampão, que poderiam encurtar a análise, foi evitado devido à possibilidade de adsorção específica do ín acetato (12) ou outros anions empregados, o que poderia influir na magnitude das cargas elétricas.

Antes de analisar os dados da figura 1 é conveniente explicar alguns detalhes sobre a obtenção dos resultados. No caso da Terra Roxa Estruturada, as determinações com $\mathrm{NaCl}$ foram feitas apenas com solução $0,2 N$, pois, com solução de concentração igual ou menor que $0,1 \mathrm{~N}$, ocorreu dispersão da argila. Ainda com relação a Terra Roxa Estruturada e cargas determinadas com $\mathrm{NaCl}$, a carga positiva no lado negativo do gráfico indica a "adsorção negativa" do aníon $\mathrm{Cl}^{-}$. Não foi feita correção para a adsorção negativa devido à dificuldade em fazê-la em presença de propriedades simultâneas de troca de catíns e de aníons. O assunto foi discutido com maiores detalhes em outro trabalho (10).

Pelo exame da figura 1, fica evidenciado que tanto a magni- 
tude das cargas positivas como a das negativas é influenciada por três fatores: $\mathrm{pH}$ da solução, concentração da solução e valência do íon trocável.

A influência do $\mathrm{pH}$ nas cargas elétricas dá-se em toda a faixa de $\mathrm{pH}$ considerada. O comportamento dos solos estudados neste trabalho torna difícil identificar a chamada carga negativa permanente, pelo menos pelas curvas de retenção de íons apresentadas. Pela forma como se apresentam as curvas, duas hipóteses podem ser levantadas com relação à carga negativa permanente: ou ela não existe (provavelmente o caso do Latossolo Roxo) ou ela é neutralizada pelo aumento da carga positiva que ocorre com o abaixamento do $\mathrm{pH}$. Para solos da União Sul Africana, Sumner e Davidtz (15), usando método de determinação de cargas elétricas similar ao deste trabalho, observaram a existência de carga negativa permanente abaixo de $\mathrm{pH} 5$ para diversos dos solos estudados, embora não para todos.

Comparando as curvas obtidas para a mesma amostra de solo com a mesma solução salina, percebe-se que as cargas elétricas são mais elevadas para maiores concentrações salinas, para um mesmo valor de $\mathrm{pH}$. Isto está de acordo com o previsto pela teoria da dupla camada, com mais um adendo importante. Aumentando-se a concentração salina, diminui-se o cancelamento mútuo das cargas positivas e negativas das duplas camadas elétricas entre si, já que a extensão da influência da carga elétrica da dupla camada no interior da fase líquida diminui com o aumento da concentração da solução. A presença concomitante de cargas positivas e negativas no solo é um indício de que as cargas estão suficientemente distanciadas para evitar neutralização mútua. Contudo, diminuindo a concentração da solução, pode-se esperar uma maior neutralização entre cargas opostas situadas umas próximas das outras na superfície do solo.

A mesma argumentação dada para explicar o efeito da concentração nas cargas elétricas pode ser aplicada para explicar o efeito da valência. Da mesma forma que o aumento na conceniração, o aumento da valência é responsável por maior carga superficial e por menor cancelamento mútuo de cargas opostas por interação de duplas camadas elétricas. As duplas camadas 
expandem-se menos no interior da fase líquida quando os íons trocáveis são divalentes do que quando eles são monovalentes. O efeito da diminuição da interação de duplas camadas com o aumento da valência parece evidente se forem comparadas as cargas positivas determinadas com o íon $\mathrm{Cl}^{-}$em presença de $\mathrm{Na}^{+}$ e de $\mathrm{Ca}^{+}$(maiores no segundo caso) ou as cargas negativas determinadas com catíon bivalente, $\mathrm{Ca}^{2+}$ ou $\mathrm{Mg}^{2+}$, em presença de $\mathrm{Cl}^{-}$ e de $\mathrm{SO}_{4}{ }^{2-}$ (maiores no segundo caso) .

Os resultados apresentados permitem levantar sérias objeções sobre o interesse da determinação da capacidade de troca de catíons para solos das regióes tropicais úmidas da maneira como é feita normalmente, isto é, com soluções salinas a concentrações bastante elevadas, muitas vezes de catíons monovalentes, e introduzindo lavagens com solventes orgânicos. Resultados assim obtidos não têm relação com as cargas elétricas que devem existir no solo em condições naturais. Provavelmente o uso de soluções diluídas de cloreto de cálcio fosse mais adequado para determinações da capacidade de troca de catíons e mesmo das cargas elétricas positivas e negativas, já que em tal caso as condições experimentais seriam mais próximas das de campo, onde os solos apresentam-se predominantemente saturados com catíons divalentes e a concentração salina da solução do solo é baixa.

DETERMINATION OF ELECTRIC CHARGES IN SOILS

\author{
SUMMARY
}

Electric charges of soils were shown to vary with concentration and $\mathrm{pH}$ of the equilibrium solution and with the valence of the counter-ions. Accordingly, in determining the electric charges of soils, total removal of excess electrolyte should be avoided and charges should be determined by measuring ion uptake under equilibrium conditions with respect to concentration and $\mathrm{pH}$. For given values of $\mathrm{pH}$ and electrolyte concentration, positive and negative charges determined with different salts for an Oxisol (Latossolo Roxo) and an Alfisol (Terra Roxa Estruturada), increased in the order $\mathrm{NaCl}<\mathrm{CaCl}_{2}<\mathrm{MgSO}_{4}$. 


\section{LITERATURA CITADA}

1. BARDSLEy, C. F. \& LANCASTER, J. D. Sulfur, In: BLACK, C. A., ed. Methods of soil analysis. Madison, Amer. Soc, Agron., 1965. p. 1102-1116. (Monogr. 9)

2. BIRRILL, K. S. \& GRADWELL, M. Ion-exchange phenomena in some soils containing amorplous mineral contituents. J. Soil Sci, $7: 130$-147, 1956.

3. BROWN, A. S. A type of AgCl elcctrode for $11 \mathrm{se}$ in dilute solutions. I. Anu. chem. Soc. $56: 646-647,1934$.

4. FIELDS, M. \& SCHOFIEIAD, R. K. Mechanisms of ion aclsorption by inorganic soil collovits. New Zcaland J. Sci. 3:563-579, 1960 .

5. FRINK, C. R. The eifect of wash solvents m cation exchange capacity mcasurements. Proc. Soil Sci. Soc. Am. 28:506-511, 1964.

6. KOLTHOFF, J. M. \& KURODA, P. K. Determination of traces of chloride. Anal. Chem. 23:1304-1306, 1951 .

7. MATMSON, S. Amionic and eationic adsorption by soil colloidal materials of varying $\mathrm{SiO}_{2} / \mathrm{Al}_{\mathrm{O}} \mathrm{O} ; \mathrm{F} \mathrm{HeO}_{3}$ ratio. In: Intern. Congress Soll Sci. 1st, Washington, 1027. Transactions. v.2, p.199-211.

\&. OKAZAKI, R.; SMITH, H.W. \& MOODIE, ( . D. Hyolrolysis and salt-retention errors in conventional cation-cxchange calacity procedutes. Soil Sci. $16: 205-209,1963$.

9. RAIJ. P. vin. Determinação de cálcio e magnćsio pelo ED'l'A em extratos ácillos de solos. Bragantia 25:317-326, 1966.

10. - Electrochemical propertics of some Brazilian soils. Cornell Universily, 1971. $144 \mathrm{fls}$. ( $\mathrm{Pl}, \mathrm{D}$. thesis)

11. \& I'EECH. M. Electrochmical properties of some Oxisols and Alfisol of the Tropics. Proc. Soil Sci. Soc. Am. 36:587-593, 1972.

12. RICH, C. I. Retroval of excess salt in cation-exchangc capacity determinations. Soil Sci. 93:87-94, 1962.

13. SCHOFEEID, R, K. Ellect of $p H$ on electric charges carried by clay particles. J. Soil Sci, 1:1-8, 1949.

14. SMITH, H. W.; MOODHE, C. D.; OKAZAKI, R. \& ELLSWORTH, $N$. I Iydrolysis and salt-retention crrors in conventional cation-exclange-capacity procedures: II. Soil Sci. 102:9.1-106, 1966.

15. SUMNER, N. E. \& DAVIDTZ, J. C. Positive and negative cliarges in some Natal soils. Soutl Air. I. agric. Scj. 8:1045-1050. 1965. 